\title{
Large-scale analysis of factors influencing nonalcoholic fatty liver disease and its relationship with liver enzymes
}

\author{
W.R. Bi ${ }^{1}$, C.Q. Yang ${ }^{2}$, Q. Shi ${ }^{1}$, Y. Xu ${ }^{1}$, C.P. Cao ${ }^{1}$, J. Ling ${ }^{1}$ and X.Y. Wang ${ }^{1}$ \\ ${ }^{1}$ Gastroenterology and Digestive Disease Institute, \\ Tongji Hospital Branch of Tongji University School of Medicine, \\ Shanghai, China \\ ${ }^{2}$ Division of Gastroenterology and Digestive Disease Institute, \\ Tongji Hospital of Tongji University School of Medicine, Shanghai, China \\ Corresponding author: C.Q. Yang \\ E-mail: cqyang@tongji.edu.cn
}

Genet. Mol. Res. 13 (3): 5880-5891 (2014)

Received December 5, 2012

Accepted March 11, 2014

Published August 7, 2014

DOI http://dx.doi.org/10.4238/2014.August.7.3

ABSTRACT. Serum liver enzyme levels are often used effectively for
the evaluation of nonalcoholic fatty liver disease (NAFLD). We aimed to
investigate the associations between serum liver enzyme levels and risks
for NAFLD in over 8000 cases in a large-scale analysis. A cross-sectional
survey with multiple stages and random samplings was performed
from May 2007 to May 2009 on 8102 workers at Tongji University. A
questionnaire was given, assessments of physical measurements, plasma
glucose, lipid profiles, and liver enzymes were made, and real-time liver
ultrasounds conducted. The prevalence of NAFLD in Tongji University
was $22.2 \%$. It was higher in males than in females (P = 0.0023). The
body mass index, waist-to-hip ratio, serum total triglycerides, serum total
cholesterol, alanine aminotransferase (ALT), aspartate aminotransferase
(AST), and gamma-glutamyl transferase (GGT) values were all higher
in the NAFLD group than in the control group. For moderate and severe
NAFLD patients, the ALT, AST and GGT values were significantly 
increased, high density lipoprotein cholesterol was decreased, and drinking much, heavy entertainment and less exercise were more prevalent $(\mathrm{P}<$ 0.001). There were strong correlations between serum liver enzyme levels and NAFLD $(\mathrm{P}<0.001)$, with GGT being a more sensitive marker for NAFLD than ALT or AST. ALT and GGT were independent predictors for NAFLD, and GGT was a better predictor than ALT for NAFLD.

Key words: Nonalcoholic fatty liver disease; Insulin resistance; Liver enzymes

\section{INTRODUCTION}

Nonalcoholic fatty liver disease (NAFLD) is the most common liver disease in adults and children worldwide. The pathological changes are similar to those for alcoholic liver disease. However, NAFLD patients have no history of excessive alcohol consumption, and the disease spectrum includes nonalcoholic fatty liver, nonalcoholic steatohepatitis (NASH), liver cirrhosis, and hepatocellular carcinoma (Raszeja-Wyszomirska et al., 2008). An estimated 20-35\% of the general population has steatosis, of which $10 \%$ will develop the more progressive NASH associated with markedly increased risk of cardiovascular- and liver-related mortality. NAFLD is a type of metabolic stress-induced liver damage, and development of NAFLD is strongly linked to components of the metabolic syndrome, including obesity, insulin resistance (IR), dyslipidemia, and type II diabetes mellitus. The recognition that NAFLD is an independent risk factor for the metabolic syndrome is a major public health concern. The cellular and molecular etiology of NAFLD is multi-factorial; genetic polymorphisms influencing NAFLD have been identified, and nutrition is a modifiable environmental factor influencing NAFLD progression.

NAFLD presents a comprehensive histological picture that results from the deposit of triglycerides in hepatocytes. It embodies a set of pathological alterations similar to those observed in alcoholic hepatopathies, but occurring in non-alcoholic individuals (Szollár, 2010). These alterations vary from simple steatosis to NASH, fibrosis, and cirrhosis (Polyzos et al., 2010). Although diagnosed all over the world, NAFLD varies in prevalence (Onyekwere et al., 2011). In the USA, a country where one fourth of the adult population is obese, steatosis is found in more than two thirds of those individuals, and in more than $90 \%$ of class III obese people (Lin et al., 2011). The actual incidence is still unknown, but is probably higher than assumed as it fails to present clinical indications, unspecific laboratory alterations including the experimental system error and, in its initial stages, liver biopsies and ultrasounds are not performed on individuals who belong to this risk group (Petta et al., 2011). Primary NAFLD is related to obesity, type II diabetes mellitus, dyslipidemia and hyperlipidemia, whereas secondary NAFLD is caused by use of some drugs, by toxins, surgeries for obesity, and total parenteral nutrition, among others (Koot et al., 2011). Increased obesity is seen in the richest social classes as well as the poorest populations, because the industrialization process has not only permitted sedentary lifestyles, but has also reduced the cost of food, making foods with high energy and fat (especially saturated fat) content more accessible (Catalano et al., 2011).

The first stages of this disease lack clinical signs, and it is not necessarily associated with clearly defined diseases such as those observed in energy and protein malnutrition (Ferreira et al., 2011). The aims of the present study were to investigate the pathogenic factors for NAFLD 
and the value of serum liver enzymes in its diagnosis, to provide evidence for the etiology of the disease, and to allow the establishment of an effective basis for prevention and control measures. Weight loss through diet and exercise is the primary recommendation in the clinical management of NAFLD. The application of systems biology to the identification of NAFLD biomarkers and factors involved in NAFLD progression is an area of promising research. Racial, ethnic, environmental, and behavioral models are also reviewed.

\section{MATERIAL AND METHODS}

\section{Patient selection and study design}

A total of 8102 workers at Tongji University were enrolled from May 2007 to May 2009. The subjects comprised 4,821 males (59.5\%) and 3281 females (40.5\%), with a mean age of $41.5 \pm 2.7$ years. All subjects were of the Han ethnic group. Three hundred and fifty random male and female cases that upon physical examination did not have NAFLD, were used as the control group. The study was approved by the Ethics Committee of Tongji University, and written informed consent was obtained from all subjects.

\section{Diagnosis standards for NAFLD}

The Diagnostic Criteria for NAFLD formulated by the Fatty Liver and Alcoholic Liver Disease Study Group of the Chinese Society of Hepatology and the Chinese Medical Association (Chinese Medical Association Hepatopathy Acad., 2010) were used in this study. The details were as follows: 1) no history of drinking or drinking alcohol equivalent to $<140 \mathrm{~g} /$ week for men, or $<70 \mathrm{~g}$ /week for women; 2) liver imaging meeting the diagnostic criteria for diffuse fatty liver, but with no other measurable factors; and/or 3) metabolic syndrome patients with unexplained increases in serum alanine aminotransferase (ALT) and/or (AST) and gamma-glutamyl transferase (GGT) levels that had continued for 6 months. After weight loss and IR improvement, patients with subsequent improvement or recovery from fatty liver (diagnosed by an abnormal enzyme spectrum and fatty liver imaging) were diagnosed as having NAFLD.

\section{Exclusion criteria}

Patients with the following conditions were excluded: a history of drinking alcohol and coffee; those with various types of viral hepatitis or familial hyperlipidemia (HLP); those being treated with drugs that can increase ALT, AST and GGT, also antihypertensive drugs, immunosuppressive agents; those with hormone, heart, brain, or kidney diseases; those with liver disease, storage disease, acute or temporary illness, autoimmune liver disease, Wilson disease, $\alpha-1$ antitrypsin deficiency, malignant liver tumors, infections and biliary tract diseases (Bondini et al., 2007).

\section{Variable definitions and diagnostic criteria}

The diagnostic criteria were as follows: smoking more than one cigarette a day for $\geq 6$ months; drinking at least once a week for $\geq 6$ months. Alcohol consumption was calculated using the following formula: ethanol amount $(\mathrm{g} / \mathrm{week})=($ beer volume + white spirit volume + fruit 
wine volume + rice wine volume) $\mathrm{x}$ alcohol degree $\mathrm{x} 0.8$ (Shi et al., 2009). Hyperuricemia was defined as serum uric acid levels of $>417 \mu \mathrm{M}$ in males or $>357 \mu \mathrm{M}$ in females (Vuppalanchi and Chalasani, 2009). Dyslipidemia was defined as serum total cholesterol (TC) of $\geq 5.69 \mathrm{mM}$ and/or serum triglyceride (TG) of $\geq 1.70 \mathrm{mM}$ (Gomercić et al., 2009). Abnormal high-density lipoprotein cholesterol (HDL-C) was defined as HDL-C of $<1.0 \mathrm{mM}$. High blood sugar was defined as fasting plasma glucose (FPG) of $\geq 6.10 \mathrm{mM}$. Hypertension was defined as systolic blood pressure of $\geq 140 \mathrm{mmHg}$ and/or diastolic blood pressure of $\geq 90 \mathrm{mmHg}$. Obesity was defined as a waistto-hip ratio (WHR; waistline:hip circumference) of $\geq 1.0$ in males and $\geq 0.85$ in females, and/or a body mass index (BMI) of $>25 \mathrm{~kg} / \mathrm{m}^{2}$. Serum liver enzyme abnormalities were defined as ALT or AST of $>40 \mathrm{U} / \mathrm{L}$ and GGT of $>50 \mathrm{U} / \mathrm{L}$ in males and $\geq 25 \mathrm{U} / \mathrm{L}$ in females (Halfon et al., 2008).

\section{General conditions}

The subjects were surveyed with a questionnaire that included the following details: drinking history and drinking amount, retrospective dietary history, family economic status, obesity and weight change history, smoking history, degree of nervousness, entertainment frequency, degree of physical exercise, disease history, level of tension, history of present illness, past medical history including hepatitis and other chronic liver diseases, family disease history. Height, weight, waistline, hip circumference, and blood pressure were all measured. The questionnaires were completed face-to-face by trained investigators.

\section{Physiochemical index examination}

Physiochemical tests were performed using methods recommended by the Chinese Society of Laboratory Medicine and the Chinese Medical Association. An OLYMPUS AU400 automatic biochemical analyzer was used for routine physiochemical tests, including TC, TG, HDL-C, LDL-C, ALT, AST, GGT, and FPG measurements, and hepatitis virus tests (HBsAg, anti-HBs, $\mathrm{HBeAg}$, anti-HBe, anti-HBc, anti-HAV-IgM, anti-HCV-IgG) were performed with reagents from Shanghai Kehua Bioengineering Co. Ltd. (KHB). Blood pressure was measured using a desktop sphygmomanometer as follows: after $5 \mathrm{~min}$ of rest in a seated position, the blood pressure of the right upper arm was measured three times and the average value calculated.

\section{Ultrasound examination}

A Logiq 500Pro color B ultrasound system (General Electric, USA; convex array probe; probe frequency $3.5 \mathrm{MHz}$ ) was used. Evaluations were performed and graded as mild, moderate, or severe. Mild NAFLD was defined as follows: in the near field, fine spots on two-thirds of the liver and the echo was lightly enhanced; in the far field, the echo from one-third of the liver was slightly weakened, and the echo contrast between the liver and kidney was slightly strengthened. Moderate NAFLD was recognized as follows: the liver was normal in shape and size; one-half of the liver in the near field showed fine spots with echo enhancement, and one-half of the liver in the far field showed sparse spots with echo attenuation; the liver showed decreased and thinner tubules; the light band of the diaphragm was unclear; the echo contrast between the liver and kidney was significantly increased. Severe NAFLD was as follows: the liver was full in shape and increased in size; the lower edges of the left and right hepatic lobes were obtuse; one-third of 
the liver in the near field presented fine spots and the echo was significantly enhanced; two-thirds of the liver in the far field presented sparse spots and the echo was attenuated; the tubules of the liver were indistinguishable; the light band of the diaphragm was unclear, and the echo contrast between the liver and kidney was significantly increased.

\section{Quality control}

The investigators were all from the Department of Physical Examination of Tongji Hospital, Tongji University. After systematic training, the investigators were able to master the standard operations in which the completed questionnaires were checked every day and imperfect questionnaires were rejected in a timely manner, and to perform periodic repairs on the equipment systems used for the body examinations. For quality control, $5 \%$ of the questionnaires, blood samples, and B ultrasound results were selected for review, and a kappa analysis confirmed that the results were good.

\section{Statistical analysis}

The sources of the EPIDATA 3.0 software were used to establish a database and SPSS 13.0 was used for the statistical analyses. The data are reported as means \pm SD. For intergroup comparisons, a $t$-test was used for two groups, and analysis of variance for multiple groups. A bilateral Fisher test was used for frequency comparisons, and the $\chi^{2}$ test was used to evaluate frequency variations. A K-S test was performed for normally distributed data, a Pearson analysis was used for univariate analyses, and a logistic regression analysis was carried out for multivariate analyses. $\mathrm{P}$ values $<0.05$ and $<0.0001$ were considered to be significant and highly significant, respectively.

\section{RESULTS}

\section{NAFLD prevalence}

Among the 8102 subjects, 1801 were confirmed to have NAFLD (22.2\%), 1093 males $(60.7 \%)$ and 708 females $(39.3 \%)$. The prevalence was higher in males than in females $\left(\chi^{2}=10.52\right.$, $\mathrm{P}=0.0002)$. The mean ages were $43.5 \pm 2.7$ years for males and $40.1 \pm 1.8$ years for females. The grades were as follows: 781 mild NAFLD cases $(9.6 \%)$, comprising 461 males $(5.7 \%)$ and 320 females (3.9\%); 645 moderate NAFLD cases (8.0\%), comprising 389 males (4.8\%) and 256 females (3.2\%); and 375 severe NAFLD cases (4.6\%), comprising 243 males (3.0\%) and 132 females (1.6\%).

\section{Univariate analyses between NAFLD patients and control subjects}

The BMI, WHR, TG, TC, ALT, AST, and GGT values were all higher in the NAFLD group than in the control group. For moderate and severe NAFLD patients, the ALT, AST and GGT were significantly increased, HDL-C was decreased, and drinking much, heavy entertainment and less exercise were more prevalent $(\mathrm{P}<0.001)$. Furthermore, as the severity of NAFLD increased, the BMI, WHR, TG, TC, LDL-C, AST, ALT, and GGT values showed an increasing trend, while there were no differences in age, family history, smoking, blood pressure, or FPG $(\mathrm{P}>0.05)$. The data are shown in Table 1, Figure 1, and Figure 2. 
Factors influencing nonalcoholic fatty liver disease

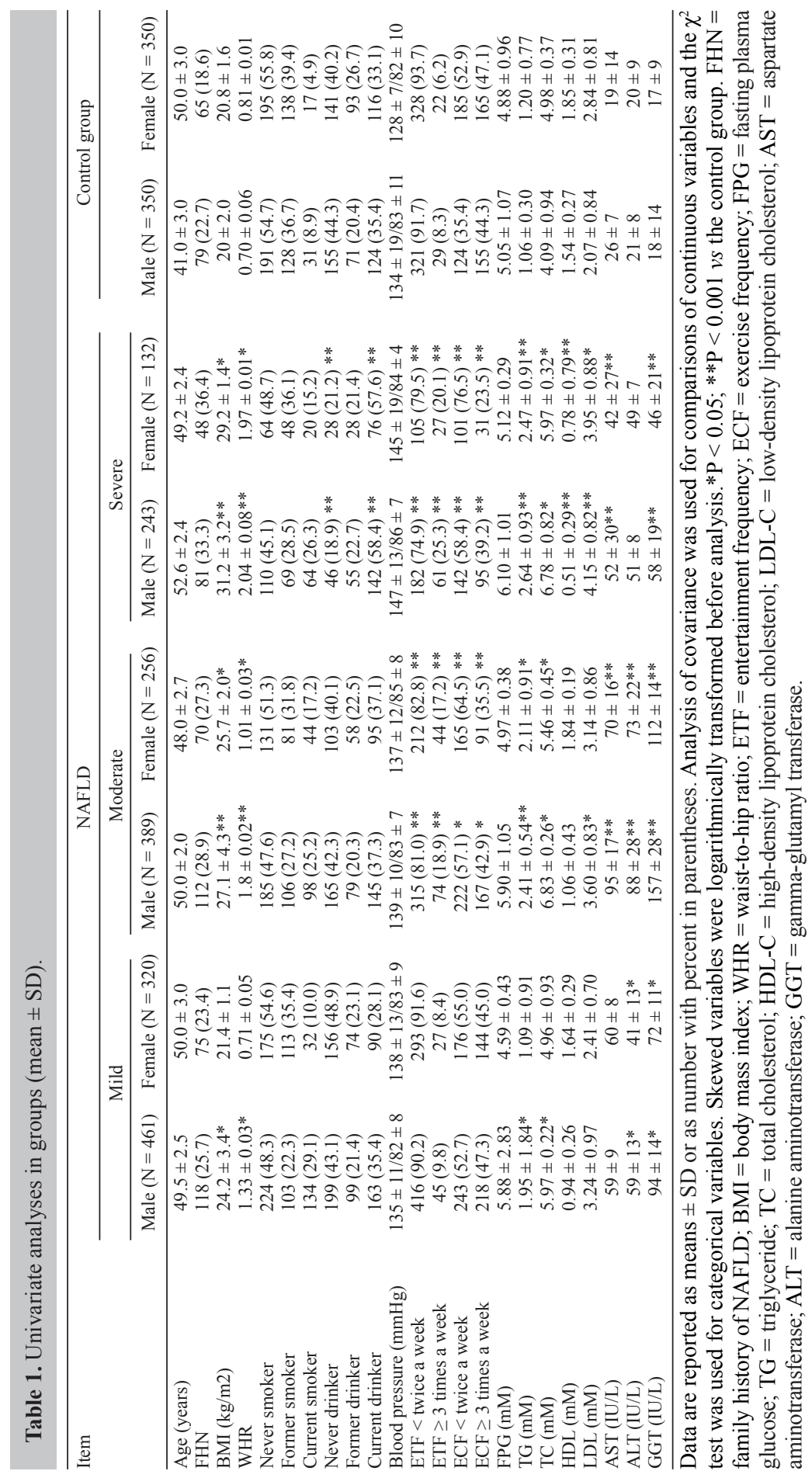




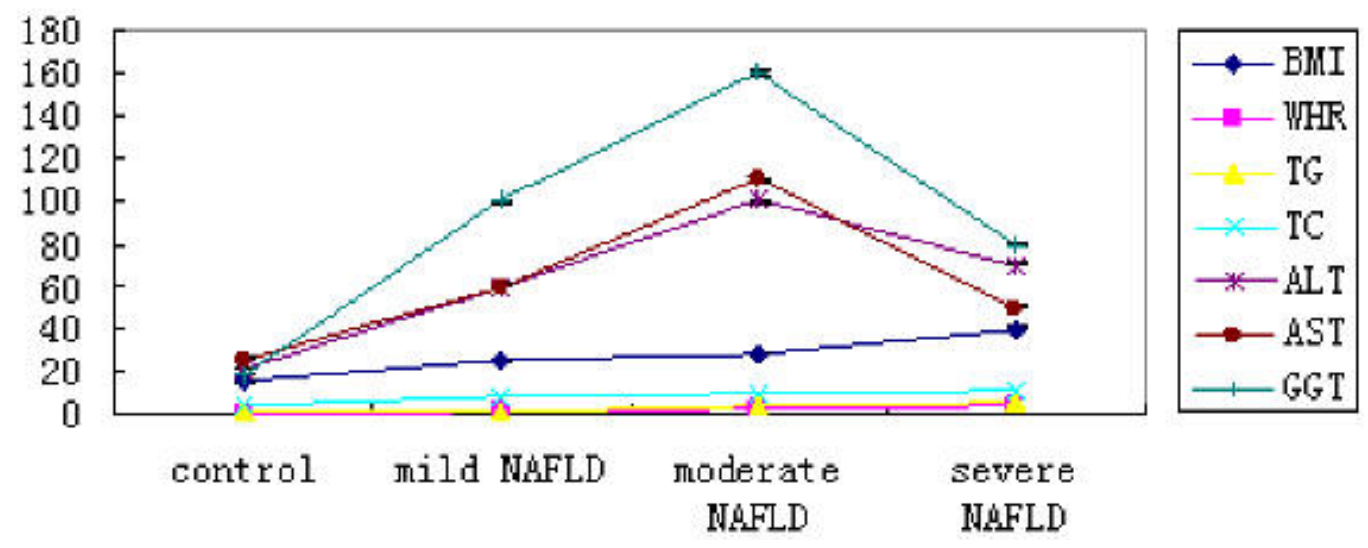

Figure 1. Analysis of BMI, WHR, TC, TG, ALT, AST, GGT in different male groups. For abbreviations, see legend to Table 1.

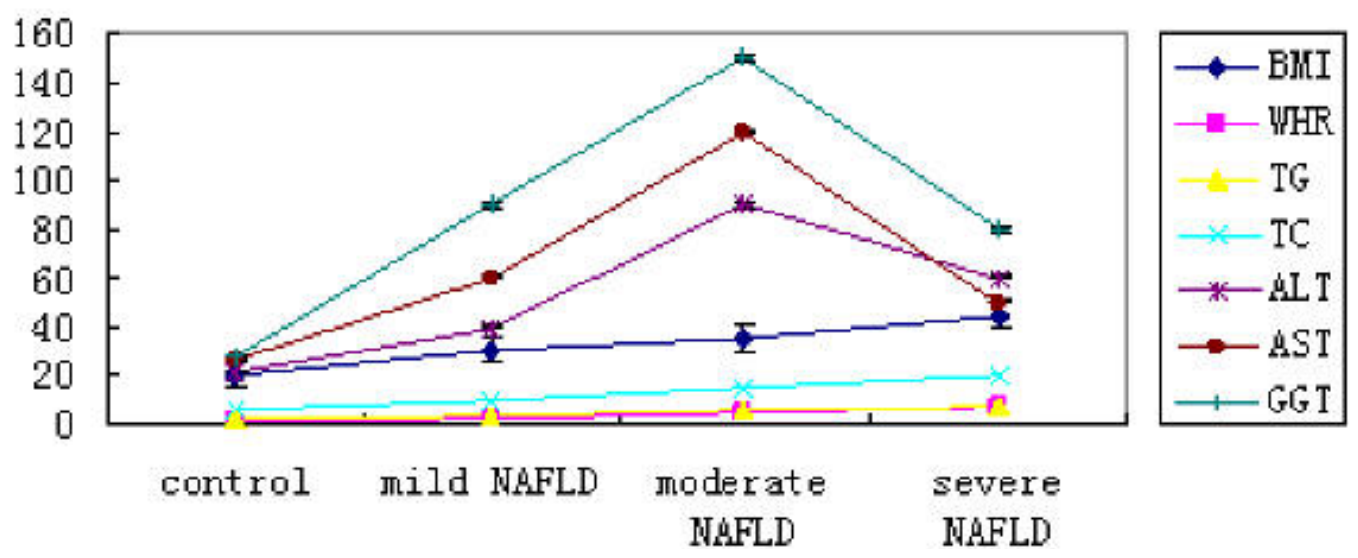

Figure 2. Analysis of BMI, WHR, TC, TG, ALT, AST, GGT in different female groups. For abbreviations, see legend to Table 1.

\section{Correlations between serum liver enzymes and NAFLD}

The prevalence rates and relative risks of metabolic syndrome were calculated by the serum ALT/GGT quartiles in the total study population, and separately in males and females. The first ALT/GGT quartile served as the reference group. Logistic regression analyses were applied with different adjustment models, and the risks are presented as the odds ratio (95\% confidence interval). Unadjusted group served as Model 1; adjusted for age, gender, family history of NAFLD, smoking and drinking status, leisure-time activity and BMI served as Model 2; Model 2 plus adjustment for serum GGT (for ALT) or ALT (for GGT) served as Model 3. We saw that both ALT and GGT were significantly associated with NAFLD in Model 3 and, furthermore, that GGT is a more sensitive marker for NAFLD than ALT and AST. The data are shown in Table 2 and Figure 3. 
Table 2. Correlations between serum liver enzymes and NAFLD.

\begin{tabular}{|c|c|c|c|}
\hline \multicolumn{4}{|l|}{ Quartiles } \\
\hline ALT (IU/L) & 1 & 2 & 3 \\
\hline \multicolumn{4}{|c|}{ Cases/number at risk (\%) } \\
\hline Total $(\mathrm{N}=8102)$ & $818 / 2622(31.2)$ & $1144 / 2778(41.2)$ & $1659 / 2702(61.4)^{* *}$ \\
\hline Male $(\mathrm{N}=4821)$ & $553 / 1502(36.8)$ & $755 / 1670(45.2)$ & $1099 / 1649(66.7)^{* *}$ \\
\hline Female $(\mathrm{N}=3281)$ & $284 / 1012(28.1)$ & $350 / 1121(31.2)$ & $629 / 1148(54.8)^{* *}$ \\
\hline \multicolumn{4}{|l|}{ Odds ratios $(95 \% \mathrm{CI})$} \\
\hline \multicolumn{4}{|l|}{ Model 1} \\
\hline Total & 1.00 & $1.31(1.13-1.55)$ & $3.31(2.81-3.86)^{* *}$ \\
\hline Male & 1.00 & $1.27(1.04-1.54)$ & $3.21(2.59-3.90)^{* *}$ \\
\hline Female & 1.00 & $1.21(0.91-1.59)$ & $3.15(2.41-4.03)^{* *}$ \\
\hline \multicolumn{4}{|l|}{ Model 2} \\
\hline Total & 1.00 & $1.54(1.26-1.85)$ & $3.29(2.64-4.03)$ \\
\hline Male & 1.00 & $1.83(1.42-2.34)$ & $3.98(2.97-5.18)^{* *}$ \\
\hline Female & 1.00 & $1.15(0.83-1.58)$ & $2.41(1.78-3.17)^{* *}$ \\
\hline \multicolumn{4}{|l|}{ Model 3} \\
\hline Total & 1.00 & $1.32(1.05-1.60)$ & $1.48(1.21-1.86)$ \\
\hline Male & 1.00 & $1.50(1.16-1.99)$ & $1.63(1.14-2.27)^{* *}$ \\
\hline Female & 1.00 & $1.01(0.72-1.43)$ & $1.24(0.81-1.86)^{* *}$ \\
\hline$\overline{\text { GGT (IU/L) }}$ & 1 & 2 & 3 \\
\hline \multicolumn{4}{|c|}{ Cases/number at risk (\%) } \\
\hline Total $(\mathrm{N}=8102)$ & $591 / 2627(22.5)$ & $1163 / 2775(41.9)$ & $1728 / 2700(64.0)^{* *}$ \\
\hline Male $(\mathrm{N}=4821)$ & $318 / 1402(22.7)$ & $588 / 1720(34.2)$ & $1186 / 1699(69.8)^{* *}$ \\
\hline Female $(\mathrm{N}=3281)$ & $265 / 1112(23.8)$ & $494 / 1071(46.1)$ & $623 / 1098(56.7)^{* *}$ \\
\hline \multicolumn{4}{|l|}{ Odds ratios $(95 \% \mathrm{CI})$} \\
\hline \multicolumn{4}{|l|}{ Model 1} \\
\hline Total & 1.00 & $2.32(1.96-2.73)$ & $5.68(4.78-6.77)^{* *}$ \\
\hline Male & 1.00 & $2.67(2.18-3.29)$ & $6.87(5.55-8.52) * *$ \\
\hline Female & 1.00 & $1.71(1.29-2.26)$ & $4.21(3.18-5.53) * *$ \\
\hline \multicolumn{4}{|l|}{ Model 2} \\
\hline Total & 1.00 & $1.95(1.60-2.38)$ & $4.36(3.62-5.21)^{* *}$ \\
\hline Male & 1.00 & $2.17(1.72-2.73)$ & $4.80(3.81-6.13)^{* *}$ \\
\hline Female & 1.00 & $1.49(1.07-2.06)$ & $3.47(2.51-4.78) * *$ \\
\hline \multicolumn{4}{|l|}{ Model 3} \\
\hline Total & 1.00 & $1.58(1.32-1.95)$ & $2.45(1.96-3.09)^{* *}$ \\
\hline Male & 1.00 & $1.79(1.38-2.27)$ & $2.42(1.82-3.23) * *$ \\
\hline Female & 1.00 & $1.23(0.88-1.73)$ & $2.31(1.58-3.42)^{* *}$ \\
\hline
\end{tabular}

Prevalence rates and relative risks of metabolic syndrome were calculated by the serum ALT/GGT quartiles in the total study population, males and females, respectively. The first ALT/GGT quartile served as the reference group. Logistic regression analyses were applied with different adjustment models and the risks are presented as the odds ratio ( $95 \%$ confidence interval). Model $1=$ unadjusted. Model $2=$ adjusted for age, gender, family history of NAFLD, smoking and drinking status, leisure-time activity and BMI. Model $3=$ model 2 plus adjustment for serum GGT (for ALT) or ALT (for GGT). **P $<0.001$ s the control group.

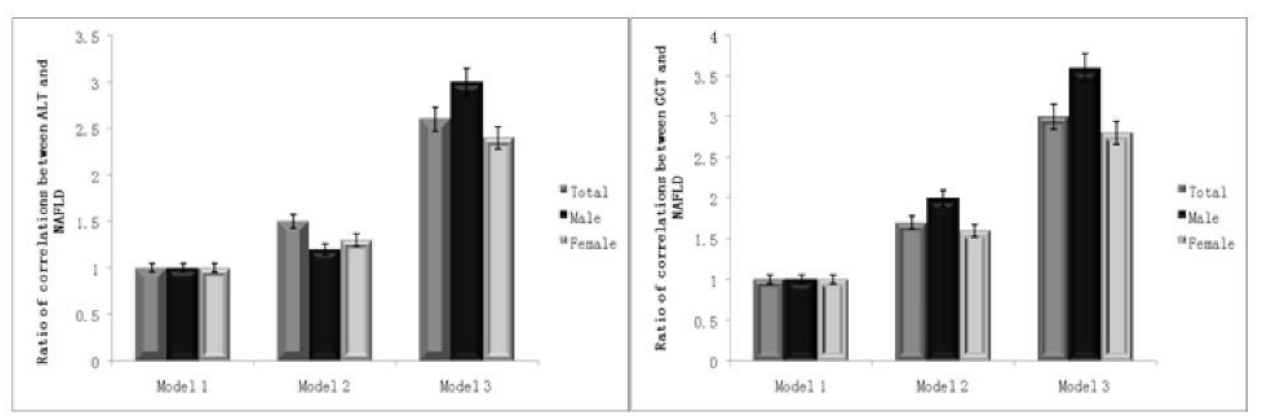

Figure 3. Correlations between ALT/GGT and NAFLD. 


\section{DISCUSSION}

We examined a population of 8102 subjects, accounting for $3.1 \%$ of all University faculty members in Shanghai. The findings showed that the prevalence of NAFLD in Tongji University was $22.2 \%$, and the average age of the affected subjects was $42.7 \pm 2.9$. This prevalence rate was consistent with those in China (20-33\%) and Asia (12-24\%) (Barshop et al., 2008), but higher than those in Shanghai, Guangzhou, Hong Kong, and other developed regions in China (15\%), and reached the prevalence rate of NAFLD in Western countries (20$30 \%$ ) (Byrne et al., 2009). The prevalence of NAFLD was higher in males than in females, and smoking, drinking, entertainment frequency, BMI, WHR, TG, TC and LDL-C were also higher in males than in females in the total surveyed population $(\mathrm{P}=0.0023)$. The possible reasons are as follows: University staffs have lifestyles that are more sedentary, males are more likely to eat out or eat an unbalanced diet, resulting in higher rates of obesity and high blood pressure, and females are more concerned about their physical appearance and obtain protection from their estrogen. All of these aspects require further investigation with a large-scale, multicenter, randomized controlled clinical trial.

In recent years, the prevalence of NAFLD has increased. NAFLD is a factor in metabolic syndrome, and is taken as a manifestation of metabolic syndrome in the liver. Obesity, type II diabetes mellitus, HLP and metabolic syndrome lead to higher risks for NAFLD, NASH and liver cirrhosis in about one-half of the global population (Petersen et al., 2010). In view of the strong correlation between IR and NAFLD, improving IR may reduce liver fat accumulation and liver cell injury in NAFLD patients (de Andrade et al., 2008). It has been reported that there are positive correlations between serum ALT/GGT and FPG, serum insulin, and WHR in NAFLD patients (Pillai and Rinella, 2009). ALT and GGT are considered independent predictors of the incidence of metabolic syndrome, diabetes mellitus, and coronary heart disease in NAFLD patients within 5-10 years (Musso et al., 2011). Serum ALT is an enzyme that plays a catalytic role in transamination in the body, and mainly functions in the cytoplasm of liver cells. The activity of ALT is 3000 times higher in liver cells than in serum. Therefore, ALT derived from liver cells and released into the blood can significantly enhance the serum level of ALT (Musso et al., 2011). As a result, ALT is considered a sensitive indicator of liver cell injury and other related types of inflammation (Ekstedt et al., 2007). GGT is a glycoprotein containing - $\mathrm{SH}$ groups that is widely distributed in mammals and is closely related to amino acid transport and detoxification in the body (Seo et al., 2008). Since GGT is thought to be an important antioxidant, an increased GGT level is considered to reflect an increase in oxidative stress in the body and more GSH transfer into the cells (Jung et al., 2008). Our study revealed that ALT and GGT are both independent predictors for NAFLD, and that GGT was a better predictor that ALT because it reflected hepatic steatosis, injury and fibrosis.

In developed Western countries, NAFLD is the most common cause of abnormal enzyme indices related to liver function and chronic liver disease. In diagnosed NAFLD patients, biochemical changes involving increased serum ALT and GGT are commonly observed, and have been regarded as predictors for NAFLD, or as markers linked with other markers to establish a formula for predicting NAFLD and progressive fibrosis of the liver (de Alwis and Day, 2008). In the present study, the proportions of NAFLD patients with increased serum ALT and GGT levels were similar to those in a previous study (de Silva and Dassanayake, 2009). It was also found that serum enzyme levels were significantly increased in mild and moderate NAFLD 
patients. The histological differences between mild to moderate and severe NAFLD presented as different severities of liver inflammation, suggesting that increased ALT and GGT may be important biochemical features of NAFLD patients in China (Wang et al., 2010).

The results revealed that increased liver enzymes were not correlated with severe NAFLD, indicating that elevated serum liver enzymes in NAFLD may simply reflect the existence of liver inflammation rather than the severity of steatosis and fibrosis of the liver. The present diagnostic system for NAFLD involves the common clinical manifestations of NAFLD and seems to be of more practical value. The system can be improved by combining it with ethnic and regional characteristics and pathological features.

Among all the variables examined in this study, serum ALT and GGT can be used for the prediction of NAFLD, but their accuracies are not high, based on age, gender, BMI, and WHR. A previous study demonstrated that serum levels of ALT and GGT gradually decrease with the progression of liver fibrosis (Kim et al., 2008). Serum ALT and GGT seem to be preferable for the assessment of liver inflammation rather than liver steatosis and fibrosis. Therefore, we should combine serum ALT and GGT with biochemical markers and demographic characteristics, using a non-invasive method to harvest comprehensive information for NAFLD. Taken together, these findings show that there is an intact disease spectrum for adult NAFLD in China, but that most cases are still in the subclinical state. These results also suggest that serum ALT and GGT levels have a certain effect on the diagnosis of adult NAFLD in China, and can reflect the degree of liver inflammation to some extent in young NAFLD patients or those with mild liver fibrosis (Lee et al., 2008). After excluding other reasons that can increase serum liver enzyme levels, serum ALT and GGT levels that are three times higher than their normal ranges can indicate the presence of NAFLD. Because some NAFLD have used liver-protecting and transaminase-lowering drugs in fact so that their serum ALT and GGT levels were normal. Although be excluded, they were real and neglected patients. Overall, NAFLD is closely related to behavior and lifestyle, such as drinking, eating fast food, frequent entertainment, and less exercise in patients who have obvious fibrosis of the liver (Fierbinteanu-Braticevici et al., 2010).

In this study, over $50 \%$ of the NAFLD patients presented abnormal serum liver enzymes. Increased WHR, TC, and TG are risk factors for abnormal liver enzymes and are components of metabolic syndrome. As the number of components of metabolic syndrome increases, there is an increased risk for abnormal serum liver enzymes, suggesting that the coexistence of multiple metabolic disorders may reflect the existence of serious liver damage, steatosis and fibrosis (Kollerits et al., 2010). We performed a cross-sectional survey regarding the correlations between serum liver enzymes and NAFLD, and found that the detection rate of metabolic syndrome was higher in NAFLD patients than in normal subjects, and that nearly one-half of the NAFLD patients presented abnormal serum liver enzymes. The dose-effect relationships between serum liver enzymes and NAFLD require further studies, and prospective research about the predictive effects of serum liver enzymes for NAFLD should be performed as well as analyses of the clinical significance of treatments for abnormal serum liver enzymes in the protection against and management of NAFLD (Kashyap et al., 2009).

The prevalence of NAFLD increased in parallel with the gradual increases in the ALT and GGT quartiles. The first quartile of ALT served as the reference, and the relative risk for NAFLD was increased in the higher ALT quartiles. However, no statistically significant differences were found in the odds ratios and relative risk changes with the higher ALT quartiles. The odds ratios in models with GGT as a predictor were greater than in those with ALT as a predic- 
tor. IR is an important factor for NAFLD, but it is not the only one. This indicates that an association exists between serum liver enzymes and NAFLD that is independent of IR (Zhu et al., 2008). Furthermore, GGT is a more sensitive marker for NAFLD than ALT. It has been reported that immunohistochemical staining can prove the existence of GGT expression in coronary plaques, suggesting that GGT directly participates in LDL oxidation in plaques (Lizardi-Cervera et al., 2006). There are two possible explanations for the potentially stronger association of GGT with NAFLD compared with that for ALT. First, both ALT and GGT are biomarkers of liver fat, but GGT may simply be the better marker. In light of the paucity of relevant evidence as to which liver enzyme better reflects liver fat content, this explanation cannot be ruled out. However, GGT is present on the surface of most cell types and is highly active in other organs, such as the kidney and pancreas. Therefore, ALT is considered a more liver-specific marker than GGT. GGT is the enzyme responsible for the extracellular catabolism of the antioxidant glutathione, and may be linked to greater oxidative stress, because oxidative stress has been implicated in IR. Our results showed that increased serum liver enzyme levels were significantly correlated with NAFLD, and that IR may be one of the main mechanisms. Compared with ALT, GGT is a better predictor for NAFLD. Based on the cross-sectional correlation analyses in our study population, future studies could focus on developing diagnostic models for NAFLD with better sensitivity and specificity that relatively accurately reflect the severity of fat deposition in a quantitative manner, and are easy to operate at low cost. We hope to improve clinical diagnostic criteria for NAFLD using multivariate noninvasive methods to provide better clinical communication in the future. To further clarify the mechanism of NAFLD, a long-term, large-scale, prospective observation is required, or even interventional research on the pathophysiological factors influencing the formation and development of NAFLD.

\section{ACKNOWLEDGMENTS}

We are grateful to Prof. Guo-Tong Xu and Prof. Jue Li for histological evaluations, and to Dr. Shu-Chang Xu for technical assistance. Research supported by a grant from the National Natural Science Foundation of China (\#81070343) and the Shanghai Innovation Program (\#12431901002).

\section{REFERENCES}

Barshop NJ, Sirlin CB, Schwimmer JB and Lavine JE (2008). Review article: epidemiology, pathogenesis and potential treatments of paediatric non-alcoholic fatty liver disease. Aliment. Pharmacol. Ther. 28: 13-24.

Bondini S, Kleiner DE, Goodman ZD, Gramlich T, et al. (2007). Pathologic assessment of non-alcoholic fatty liver disease. Clin. Liver Dis. 11: 17-23.

Byrne CD, Olufadi R, Bruce KD, Cagampang FR, et al. (2009). Metabolic disturbances in non-alcoholic fatty liver disease. Clin. Sci. 116: 539-564.

Catalano D, Trovato GM, Martines GF, Pirri C, et al. (2011). Renal function and severity of bright liver. Relationship with insulin resistance, intrarenal resistive index, and glomerular filtration rate. Hepatol. Int. 5: 822-829.

Chinese Medical Association Hepatopathy Acad. (2010). Branch Hepatic Adipose Infiltration and Alcoholic Fatty Liver Disease Method Group. Nonalcoholic fatty liver disease diagnosis and therapy manual (2010 revised edition). Chin. J. Hepatol. 18: 163-166.

de Alwis NM and Day CP (2008). Non-alcoholic fatty liver disease: the mist gradually clears. J. Hepatol. 48 (Suppl 1): S104-S112.

de Andrade AR, Cotrim HP, Alves E, Soares D, et al. (2008). Nonalcoholic fatty liver disease in severely obese individuals: 
the influence of bariatric surgery. Ann. Hepatol. 7: 364-368.

de Silva HJ and Dassanayake AS (2009). Non-alcoholic fatty liver disease: confronting the global epidemic requires better awareness. J. Gastroenterol. Hepatol. 24: 1705-1707.

Ekstedt M, Franzen LE, Mathiesen UL, Holmqvist M, et al. (2007). Statins in non-alcoholic fatty liver disease and chronically elevated liver enzymes: a histopathological follow-up study. J. Hepatol. 47: 135-141.

Ferreira DM, Castro RE, Machado MV, Evangelista T, et al. (2011). Apoptosis and insulin resistance in liver and peripheral tissues of morbidly obese patients is associated with different stages of non-alcoholic fatty liver disease. Diabetologia 54: 1788-1798.

Fierbinteanu-Braticevici C, Dina I, Petrisor A, Tribus L, et al. (2010). Noninvasive investigations for non alcoholic fatty liver disease and liver fibrosis. World J. Gastroenterol. 16: 4784-4791.

Gomercić M, Duvnjak M and Barsic N (2009). Ultrasonography in the diagnosis of nonalcoholic fatty liver disease. Acta Med. Croatica 63 (Suppl 3): 1-3.

Halfon P, Munteanu M and Poynard T (2008). FibroTest-ActiTest as a non-invasive marker of liver fibrosis. Gastroenterol. Clin. Biol. 32: 22-39.

Jung SH, Park HS, Kim KS, Choi WH, et al. (2008). Effect of weight loss on some serum cytokines in human obesity: increase in IL-10 after weight loss. J. Nutr. Biochem. 19: 371-375.

Kashyap SR, Diab DL, Baker AR, Yerian L, et al. (2009). Triglyceride levels and not adipokine concentrations are closely related to severity of nonalcoholic fatty liver disease in an obesity surgery cohort. Obesity 17: 1696-1701.

Kim WR, Flamm SL, Di Bisceglie AM and Bodenheimer HC (2008). Serum activity of alanine aminotransferase (ALT) as an indicator of health and disease. Hepatology 47: 1363-1370.

Kollerits B, Coassin S, Kiechl S, Hunt SC, et al. (2010). A common variant in the adiponutrin gene influences liver enzyme values. J. Med. Genet. 47: 116-119.

Koot BG, van der Baan-Slootweg OH, Tamminga-Smeulders CL, Rijcken TH, et al. (2011). Lifestyle intervention for non-alcoholic fatty liver disease: prospective cohort study of its efficacy and factors related to improvement. Arch. Dis. Child. 96: 669-674.

Lee TH, Kim WR, Benson JT, Therneau TM, et al. (2008). Serum aminotransferase activity and mortality risk in a United States community. Hepatology 47: 880-887.

Lin YC, Chou SC, Huang PT and Chiou HY (2011). Risk factors and predictors of non-alcoholic fatty liver disease in Taiwan. Ann. Hepatol. 10: 125-132.

Lizardi-Cervera J, Laparra DI, Chavez-Tapia NC, Ostos ME, et al. (2006). Prevalence of NAFLD and metabolic syndrome in asymtomatics subjects. Rev. Gastroenterol. Mex. 71: 453-459.

Musso G, Gambino R, Cassader M and Pagano G (2011). Meta-analysis: natural history of non-alcoholic fatty liver disease (NAFLD) and diagnostic accuracy of non-invasive tests for liver disease severity. Ann. Med. 43: 617-649.

Onyekwere CA, Ogbera AO and Balogun BO (2011). Non-alcoholic fatty liver disease and the metabolic syndrome in an urban hospital serving an African community. Ann. Hepatol. 10: 119-124.

Petersen KF, Dufour S, Hariri A, Nelson-Williams C, et al. (2010). Apolipoprotein C3 gene variants in nonalcoholic fatty liver disease. N. Engl. J. Med. 362: 1082-1089.

Petta S, Di Marco V, Camma C, Butera G, et al. (2011). Reliability of liver stiffness measurement in non-alcoholic fatty liver disease: the effects of body mass index. Aliment. Pharmacol. Ther. 33: 1350-1360.

Pillai AA and Rinella ME (2009). Non-alcoholic fatty liver disease: is bariatric surgery the answer? Clin. Liver Dis. 13: 689-710.

Polyzos SA, Kountouras J, Zavos C and Tsiaousi E (2010). The role of adiponectin in the pathogenesis and treatment of non-alcoholic fatty liver disease. Diabetes Obes. Metab. 12: 365-383.

Raszeja-Wyszomirska J, Lawniczak M, Marlicz W, Miezynska-Kurtycz J, et al. (2008). Non-alcoholic fatty liver diseasenew view. Pol. Merkur Lekarski. 24: 568-571.

Seo YS, Kim JH, Jo NY, Choi KM, et al. (2008). PPAR agonists treatment is effective in a nonalcoholic fatty liver disease animal model by modulating fatty-acid metabolic enzymes. J. Gastroenterol. Hepatol. 23: 102-109.

Shi JP, Xun YH, Hu CB, Zhang L, et al. (2009). Clinical and histological features of non-alcoholic fatty liver disease. Zhonghua Gan Zang. Bing Za Zhi 17: 812-816.

Szollár L (2010). Fatty liver and global cardiometabolic risk. Orv. Hetil. 151: 1946-1950.

Vuppalanchi R and Chalasani N (2009). Nonalcoholic fatty liver disease and nonalcoholic steatohepatitis: Selected practical issues in their evaluation and management. Hepatology 49: 306-317.

Wang Y, Dong J, Liu HY, Wang DQ, et al. (2010). Biochemical characteristics and risk factors in non-alcoholic fatty liver. Front Biosci. 2: 105-110.

Zhu FS, Liu S, Chen XM, Huang ZG, et al. (2008). Effects of n-3 polyunsaturated fatty acids from seal oils on nonalcoholic fatty liver disease associated with hyperlipidemia. World J. Gastroenterol. 14: 6395-6400. 